\title{
Connecting for Health: Playing with Horses as a Therapeutic Tool.
}

Annick Maujean, PhD, Population and Social Health Research Program, Griffith Health Institute, Griffith University, Australia

Elizabeth Kendall, PhD, Centre for National Research on Disability and Rehabilitation Medicine, Griffith Health Institute, Griffith University, Australia Lillan Roquet, Griffith Health Institute, Australia Tony Sharp, Youth and Family Service (Logan City) Inc., Slacks Creek, Australia Graham Pringle, State Manager (Queensland), Evolve - Keeping Kids on Track, Mt Tamborine, Australia

Correspondence should be addressed to:

Dr Annick Maujean

Population and Social Health Research Program,

Griffith Health Institute, Griffith University,

Meadowbrook Q 4131 Australia

Telephone: +61 733821295

Facsimile: +61733821414

Email: $\quad$ a.maujean@griffith.edu.au 


\begin{abstract}
This pilot study examined a brief psychological intervention using horses as a therapeutic tool to improve levels of self-esteem, self-efficacy, and facilitate the development of life skills in a group of disengaged youths $(n=16)$. Participants and their case managers were interviewed following completion of the program to explore their responses to the intervention. Interview transcripts were coded to identify participants' experiences and outcomes. Five themes emerged from these interviews, namely (1) enjoyment, (2) psychological and social benefits, (3) engagement, (4) transferrable skills, (5) mechanisms of change. The findings of this study indicate that this type of intervention may provide a viable option for youths who are disengaged from school and/or the community and who have not responded to traditional interventions.
\end{abstract}




\section{Introduction}

The belief that animal-human interactions can act as a therapeutic tool has developed in recent years into a formalised field of therapy known as animal-assisted therapy (AAT) (Burgon, 2011). AAT uses the human-animal bond as an integral part of the therapeutic process (Chandler, 2005). Compared to other animals, the horse is argued to have additional therapeutic qualities, in part because of its size and power, but also due to its inherent vulnerability as a prey animal and the unique dynamic this situation creates (Vidrine, OwenSmith, \& Faulkner, 2002).

Therapy involving horses has been referred to by many different terms, such as equine facilitated learning, equine facilitated psychotherapy, and hippotherapy. For the purpose of this study, the term equine facilitated psychotherapy (EFP) will be used. EFP is an experiential psychotherapy that engages horses as co-therapists to promote emotional growth and learning (EAGLA, 2006). Although therapeutic horse riding has been a recognized activity for many decades, particularly in the area of disability, EFP goes beyond the activity of riding and uses the task of caring for horses as a mean of establishing trust, respect, and responsibility. Programs usually involve engagement in activities that require clients to apply specific skills such as non-verbal communication, assertiveness, teamwork and relationships, with a view to building confidence and positive attitude (EAGLA, 2006).

Although EFP can involve a range of horse-related activities (e.g., riding, grooming, handling, and lunging), many programs continue to rely on serendipitous therapeutic benefit rather than planned therapeutic intervention. There is a need to investigate the role of more structured programs that build on the psychology of the horse and the translation of these principles into human social and emotional states.

Birke's (2008) study of the most common methods of interacting with horses identified a cluster of approaches which were loosely referred to as "natural horsemanship". One of the 
most thoroughly marketed and frequently used methods of natural horsemanship is that developed by Pat Parelli in the USA (Parelli Natural Horsemanship - PNH, http://www.parellinaturalhorsetraining.com). Although containing many other components, the mainstay of the PNH program is a structured sequence of seven "games" (e.g., http://www.parellinaturalhorsetraining.com/parelli-7-games-the-friendly-game/) that allow people to progressively learn the principles of interacting successfully with a horse. Each game requires consistency, clear non-verbal communication, observation, responsiveness and assertion. Humans must develop a trusting relationship with their horse by understanding how their own behaviours are perceived and by meeting the needs of the horse as determined from an equine perspective rather than a human perspective. If these structured activities are supported with observations and feedback, meaningful interpretation and deliberate translation of skills into the human context, the PNH approach can be used to furnish young people with skills that can extend beyond the equine environment.

We propose that EFP built on a structured model of natural horsemanship, rather than relying only on unstructured interactions with horses, may demonstrate therapeutic benefit. The use of the PNH approach, which we have selected to underpin this research, can enable the program to simultaneously capitalize on (1) the motivation and engagement that can be derived from horses and the rural environment, (2) transferrable social and interpersonal skills that can be derived from the process of understanding and applying horse psychology (3) psychological growth that can be derived from the process of building on experiences and performance, and (4) a sense of mastery that can be derived from the process of incrementally achieving a structured sequence of training tasks and successfully performing a novel activity.

The purpose of this paper is to report on a pilot study of a brief psychological intervention called Horse Play which was delivered within a program called Connecting4Health. The 
program was delivered by an alliance of organizations, including Griffith University, the Logan Beaudesert Health Coalition, Evolve: Keeping Kids on Track, Youth and Family Services and a PNH instructor, Lillan Roquet a Licenced 2 Junior Parelli Professional.

\section{Method}

\section{Participants}

Sixteen adolescents participated in the pilot study (11 males and 5 females), with a mean age of 15.44 years $(S D=3.12$ years; range $=12-22$ years $)$. The participants were recruited through Youth and Family Services (YFS) in Queensland, Australia. YFS is an organisation that offers a wide range of services and programs for young people, including those who have disengaged from school and are considered "at-risk". The adolescents who were recruited to the current pilot program were selected by their case managers to deliberately represent youth who had failed to respond to previous traditional interventions. No other inclusion criteria were applied to the program.

\section{Data Collection}

\section{Case manager surveys}

Immediately post-intervention, the case managers were asked five open-ended questions relating to changes (if any) in the young persons' behaviours and attitude to life. Case managers were also asked to nominate the features of the intervention they believed might be responsible for any changes and why they had attributed these changes to the program. An example open-ended question is "Have you noticed any change in the young person's attitude to life since he/she has attended the Horse Play program? (If yes, please give details)."

Finally, at six months following completion of the program, a meeting was held with the case managers to discuss key outcomes and progress of participants. At this meeting, case 
managers were asked to comment on the level of engagement of participants in school and work (if relevant).

\section{Semi-structured interviews}

Semi-structured interviews were conducted prior to and immediately following the intervention. The interview was designed to explore participants' level of motivation and expectations before taking part in the program, their perceived strengths and abilities, areas where they felt they needed to improve in their life, and general information about their friendships, school and family life. The post-intervention interview focused on participants' personal experiences while taking part in the program (i.e., what they liked and disliked about the program, personal skills/abilities they acquired during the program).

\section{The Intervention}

The intervention consisted of ten weekly sessions. The sessions began with demonstrations of natural horsemanship, followed with practice. Each participant was matched to a horse based on assessments of their internal or external orientation. The participant/horse pairs were also matched to a mentor who assisted them with their practice sessions. Their case managers also attended the sessions, but did not participate in the intervention.

At the end of each week, participants discussed the skills or abilities they had learned during the practice session. These group discussions were led by a psychologist, and included the team of mentors and the instructor. The groups enabled members to provide positive feedback to the participants and encouraged discussion about how these new skills and knowledge could be applied in their everyday lives.

Every week the young persons were asked to select one word from a list of 31 qualities (e.g., team player, communication, forgiveness, assertiveness, kindness). A discussion was then held about how they aimed to implement or practice this quality in their 
everyday life. Their experiences during the week were discussed at the beginning of the following session, when they were encouraged to describe ways in which their new skill had been useful. The list of qualities was constructed by the psychologist by reviewing the content of the PNH program and deriving all the qualities that might be triggered by the intervention. A definition of each quality was typed on an A4 page (e.g., tolerance willingness to accept behaviors and beliefs that are different from your own, although you might not agree with or approve of them).

\section{Results}

\section{Attendance and engagement in the program}

Twelve of the 16 participants completed the program. All 12 participants were enthusiastic about the program, based on case managers' reports and observations during the program. Of the four participants who did not complete the program, one female participant moved overseas after attending four sessions; another female participant had to withdraw after the first session due to personal problems; two (one male and one female) participants dropped out of the intervention program after the third session for reasons unknown.

\section{Qualitative results}

The interview transcripts were coded to identify significant statements and phrases that directly reflected participants' experiences or outcomes. The relevant pieces of text were extracted and organised into themes.

Enjoyment - The most common theme to emerge from the post-intervention interviews was that all 12 participants thoroughly enjoyed their participation and expressed more frequent positive moods following the program. For example, one participant stated that he thought, "it [Horse Play program] was awesome, I liked it, it was great". The 'enjoyment' and 'fun' described by the young persons were confirmed by their case managers who described how all participants looked forward to the sessions each week with 
anticipation and excitement. For example, one case manager stated that, "XXX [young person] has increasingly looked forward to attending 'Horse Play' each week as the course has progressed. His behaviour has changed from being agitated to expressing positive thoughts and having a 'cheerful' disposition". Other case managers referred to a "lightening" of the participant's mood, with "less depressive episodes" and an "increase in sense of humour" or a "sense of calmness". One case manager noted an "increase in the period of time for which [young person] is stable and calm". A minor, but potentially related, finding was the fact that some participants were observed to become more healthy in terms of their physical activity, eating and sleeping habits.

Psychological and social benefits of the program - Prior to taking part in the Horse Play program, participants were asked to identify the strengths/abilities and positive characteristics they believed they have. Although some participants were able to identify one positive characteristic about themselves, their statements were often qualified or tentative, e.g., "I reckon I'm a good worker" and "I think I am a good person". It was more common that participants could not identify anything positive about themselves, stating that they "didn't know" or "didn't believe" they had any positive characteristics or skills/abilities.

In contrast, during the post-intervention interviews, participants reported various skills and abilities they had learned about themselves while taking part in this program. One common example was the sense of achievement associated with overcoming fear. Two participants reported being scared of interacting with their horse at the start of the program but gradually learned to overcome their fear, "Big Sam (horse), I would never in my life think that I'd go near a horse his size"; "Well, it [fear] was still there but it wasn't holding me back so much".

Another theme that emerged during these post-intervention interviews was the social skills participants had developed while interacting with their peers and instructors/mentors 
during the program, as one participant stated, "I have learnt about being friends with people". Another participant commented that his interaction with horses has helped him develop confidence in his ability to interact with others - "because [being with] a horse is like [being with] a human, it's helping me getting along with other people now". Case managers also noticed an improvement in participants' interactions with others, including family members, as well as an increased ability to be compassionate, patient and tolerant towards others. The following anecdotal responses given by case managers illustrate this point - "the young person has demonstrated consideration for others....and has developed considerable patience when interacting with others"; "the young person has demonstrated a sense of care toward his family and acknowledges his role in the family"; "the young person recognizes the level of decision making in the house". Other social changes that were noted by case managers included increased kindness towards others, cooperation, helpful behaviour and willingness to participate with others. One case manager commented on how these changes were evident in participants' posture (e.g., "standing more upright", "increase in eye contact", "smiles more"). The result of this change was that participants were seen as being "less lonely" or "more willing to socialise with other people".

Participants reported a boost in their overall level of confidence and self-worth. One participant stated, "I really became more confident in doing what I set out to do". Another participant expressed a greater sense of value about himself, "I feel good in the way I dress, I feel good around horses, and I'm kind of still the same about people, I'm a little shyish but try not to be". Participants' case managers commented on the increasing level of confidence that the young people were gradually developing while participating in the program. This increased confidence is illustrated in the following quote from a case manager - "There has been a gradual decrease in timidity and an increase in [the young person's] level of selfconfidence. He is more willing to chat to different people". Several case managers 
commented on the fact that participants now took more pride in their achievements rather than dismissing them. They reported that participants had increased beliefs in their ability to tackle complicated problems and were able to accept compliments. Case managers described how participants left each session with a "sense of achievement and the satisfaction that goes along with that".

Engagement - Case managers observed important positive changes in participants' engagement with various aspects of their lives. This change was most pronounced in terms of their attitudes and behaviors towards attending school. For example, a case manager reported that one of the participants stated that "the [Horse Play] program has helped me go to school". This statement reflected the participant's regular attendance at school and the decrease in his negative perception of school and school personnel since attending the Horse Play program. All participants of school age returned to school during the program despite having been disengaged from school for long periods of time prior to the program. The two older participants were engaged in voluntary roles and had enlisted to become mentors in a subsequent Horse Play program.

Case managers reported an increase in the extent to which participants were engaging in other non-horse activities. This increased engagement was illustrated by the following comment made by a case manager about one of the participants, "XXX [young person] is now keen to come to other activities, before he would stay in his room for long periods of the day. He will now readily participate. He is beginning to want to experience new situations". This openness to new activities was a common observation of case managers. They described how participants had begun engaging with other sports or programs whereas they had previously been reluctant to participate. Case managers reported examples of how participants had shown an interest in similar outdoor programs, suggesting that the program had "nurtured a desire in XXX [young person]". As one case manager described, the 
program "exposed $\mathrm{XXX}$ [young person] to a new environment, the rural setting, he is developing an appetite to discover more about country living and nature". This new interest was described as the foundation for a new attitude towards life and enthusiasm, but also a willingness to commit to a course of action and an openness to new ways of doing things.

Transferrable Skills - Case managers reported that the various skills participants learned during this program were conducive to positive changes in participants' behaviors and attitudes towards life. When case managers described the general changes they had observed in participants' daily behaviors, they mirrored those that underpinned the program (e.g., greater levels of self-control, increased motivation, improved concentration and patience, and enhanced self-awareness). Case managers described the fact that participants appeared to "think more about their actions" or were "more organized in their thoughts". Given that case managers were blind to the underlying skills that were likely to be fostered by the program, this finding suggests that transfer of skills between the horse environment and the human environment may have occurred.

Mechanisms of Change - The most common response from case managers when asked to describe the potential mechanisms of change was the participant/horse connection, which was not surprising given that each participant was paired with the same horse every week (other than a few minor changes due to availability). When interviewed, participants also mentioned the connection they had formed with 'their' horse. One participant stated that what he enjoyed the most was "to play with horses and connect with 'my' horse". Another participant described his affection for 'his' horse - "half the time I was hugging Goldie (horse), half the time scratching her in the right spot...". Participants' connection with their horse was instrumental to some of the positive changes observed in these participants as illustrated by the following quote from a case manager - "a major aspect of generating 
change for XXX [young person] has been his connection with his horse. I think this has been very therapeutic for him".

Other mechanisms of change, however, revealed the significance of the context within which the program was conducted (e.g., "an engaging but peaceful environment with multiple sensory experiences"). The value of the rural environment was repeatedly noted by participants and case managers during the delivery of the program, with observations that participants physically relaxed as soon as they entered the property.

Case managers also highlighted the critical role of program staff as primary mediators through which the program impacted on participants. They observed that program staff provided unconditional guidance while participants learned the necessary skills (e.g., "positive interactions with a range of people", "cooperation with other program participants", the "time taken to make sure participants felt safe and confident", "consistent praise for achievements" and "encouragement to engage", "having positive adults around him", "being around people who were accepting of him").

Of interest, however, was the fact that case managers believed a significant mechanism of change was the nature of the program itself in that it was structured with clear tasks but involved unstructured time for reflection. It also involved gradual exposure to, and mastery of, an unfamiliar situation that presented a high level of perceived challenge for participants. Finally, case managers commented on the good matching of horses to people. They observed how the personality of the horse tended to complement the personality of the participant, which facilitated the likelihood of learning.

\section{Discussion}

Based on the interviews conducted with the participants and their case managers, there was strong evidence that the behaviours and skills learned in the program had been translated into the participants' everyday lives and they demonstrated a positive shift in their 
overall attitude towards life. The findings of this study lend support to the argument that EFP is a mechanism for providing opportunities to increase the development of life skills and improve levels of confidence and engagement in young individuals at risk of disengagement and mental illness.

There are a number of factors that may have contributed to the positive psychosocial outcomes described by these participants. Specifically, this program provided a nonjudgmental environment where participants' positive characteristics, personal strengths and abilities were highlighted and encouraged while they interacted with their horses. They also interacted with a range of people, including other participants and the team of horse facilitators, case managers, and a psychologist. Thus, the program provided a social learning environment. With its calming rural environment, it also provided a positive context within which therapeutic relationships could be established and sensitive discussions became less threatening.

Second, the Parelli training method enabled participants to become competent in horse handling and training tasks. All participants reached a level of development where their horse could achieve major tasks which would not ordinarily be expected of a horse (e.g., perform patterns of behaviour off-lead, stand on a small pallet, walk through obstacles, stop or back up in response to minor physical cues etc.). The feeling of achievement that was likely to be associated with this outcome would have contributed to the gradual development of confidence. The ability to handle such a powerful animal positioned participants as members of a small "elite" group within the population, which would also have contributed to their sense of achievement. It is not surprising that some participants began wearing equine or rural attire and sought greater involvement in rural programs or lifestyles following the program. 
According to Kersten and Thomas (2003) confidence and self-esteem can be greatly increased through the accomplishment of competently handling horse-oriented tasks. The size and power of a horse demands respect and would therefore be intimidating for most people. At the beginning of the program, some participants felt a significant level of fear and intimidation in approaching and interacting with their horse. However, during the course of the program they were able to manage and overcome their fear, which may have been a conduit to developing belief in their ability to overcome other difficulties they faced in their complicated daily lives.

Although limited by the small size of the sample, this study has provided some useful conclusions about the potential of EFP using a natural horsemanship orientation and the potential mechanisms by which benefits are likely to be realised. As noted by Lentini and Knox (2009), a great deal more work is required before EFP could become an integral component of the therapy landscape, particularly given the costly nature of equine programs and the practical constraints associated with running programs. Nevertheless, our data has suggested that this type of therapy may provide a viable option for those who have not responded to other interventions, have disengaged from all traditional institutions and are facing potentially serious consequences in the future if not assisted in some way. 


\section{References}

Birke, L. (2008). Talking about horses: Control and freedom in the world of "Natural Horsemanship". Society and Animals, 16, 107-126.

Burgon, H. L. (2011). 'Queen of the world': experiences of 'at-risk' young people participating in equine-assisted learning/therapy. Journal of Social Work Practice, 25(2), 165-183.

Chandler, C. K. (2005). Animal assisted therapy in counseling. New York: Routledge.

Equine Assisted Growth and Learning Association (EAGLA). (2006). What is EAP and EAL? Retrieved July 6, 2011, from http://www.eagla.org

Kersten, G., \& Thomas, L. (2003). Why horses? In L. Thomas and G. Kersten (Eds.), Equine-assisted mental health resource handbook. USA: Eagala Inc.

Lentini, J.A., \& Knox, M. (2009). A qualitative and quantitative review of equine facilitated Psychotherapy (EFP) with children and adolescents. The Open Complementary Medicine Journal, 1, 51-57.

Vidrine, M., Owen-Smith, P., \& Faulkner, P. (2002). Equine-facilitated group psychotherapy: Applications for therapeutic vaulting. Issues in Mental Health Nursing, 23, 587-603 